\title{
PMBM Filter With Partially Grid-Based Birth Model With Applications in Sensor Management
}

Per Boström-Rost, Daniel Axehill and Gustaf Hendeby

The self-archived postprint version of this journal article is available at Linköping University Institutional Repository (DiVA):

http://urn.kb.se/resolve?urn=urn:nbn:se:liu:diva-182957

N.B.: When citing this work, cite the original publication.

Boström-Rost, P., Axehill, D., Hendeby, G., (2022), PMBM Filter With Partially Grid-Based Birth Model With Applications in Sensor Management, IEEE Transactions on Aerospace and Electronic Systems, 58(1), 530-540. https://doi.org/10.1109/taes.2021.3103255

Original publication available at:

https://doi.org/10.1109/taes.2021.3103255

Copyright: Institute of Electrical and Electronics Engineers

http://www.ieee.org/index.html

(C)2022 IEEE. Personal use of this material is permitted. However, permission to reprint/republish this material for advertising or promotional purposes or for creating new collective works for resale or redistribution to servers or lists, or to reuse any copyrighted component of this work in other works must be obtained from the IEEE. 


\title{
PMBM filter with partially grid-based birth model with applications in sensor management
}

\author{
Per Boström-Rost, Daniel Axehill, Senior Member, IEEE, Gustaf Hendeby, Senior Member, IEEE \\ Dept. of Electrical Engineering, Linköping University \\ Linköping, Sweden
}

\begin{abstract}
This paper introduces a Poisson multi-Bernoulli mixture (PMBM) filter in which the intensities of target birth and undetected targets are grid-based. A simplified version of the Rao-Blackwellized point mass filter is used to predict the intensity of undetected targets and to initialize tracks of targets detected for the first time. The grid approximation can efficiently represent intensities with abrupt changes with relatively few grid points compared to the number of Gaussian components needed in conventional PMBM implementations. This is beneficial in scenarios where the sensor's field of view is limited. The proposed method is illustrated in a sensor management setting where trajectories of sensors with limited fields of view are controlled to search for and track the targets in a region of interest.
\end{abstract}

Index Terms-Poisson multi-Bernoulli mixture filter, multitarget tracking, sensor management, Rao-Blackwellized point mass filter

\section{INTRODUCTION}

This paper considers multi-target tracking problems where the sensor's field of view is much smaller than the region of interest. In such scenarios, it is not only of interest to estimate the states of detected targets, but also to estimate where undetected targets may be located. This can be done by combining information about areas that have been covered by the sensor with prior knowledge about where targets are likely to appear. The problem is complicated as the number of targets is both unknown and time-varying, there are misdetections, false alarms, and unknown measurement origins.

The random finite set (RFS) framework enables a Bayesian approach to the considered problem [1]. Several RFS-based methods to estimate the multi-target posterior density have been proposed. Examples of these include the probability hypothesis density (PHD) filter [2], the generalized labeled multi-Bernoulli (GLMB) filter [3, 4], and the Poisson multiBernoulli mixture (PMBM) filter [5]. The PMBM filter assumes that the multi-target density consists of a union of a Poisson point process (PPP) and a multi-Bernoulli mixture (MBM) [5]. The MBM component considers all possible data association hypotheses and can, e.g., be implemented using a track-oriented multiple hypotheses tracking (MHT) formulation [6]. The PPP explicitly models the intensity of undetected targets, thereby indicating where it is likely to find

This work was supported in part by the Wallenberg AI, Autonomous Systems and Software Program (WASP) funded by the Knut and Alice Wallenberg Foundation; in part by the Industry Excellence Center LINKSIC funded by The Swedish Governmental Agency for Innovation Systems (VINNOVA); and in part by Saab AB. new targets [5], which is key information in Bayesian searchand-track sensor management.

In PMBM filter implementations, the birth intensity and intensity of undetected targets are commonly modeled as a single Gaussian or a Gaussian mixture [6-8]. While this approach is suitable when the sensor's field of view covers most of the region of interest, it is potentially inefficient if the field of view is small or if the birth intensity is uniform in the region of interest. Although a Gaussian mixture can approximate any intensity, a large number of components may be necessary to approximate a uniform density or to obtain sharp edges around regions that have recently been visited by the sensor. One alternative could be to apply the ideas of [9]; start with a small number of components and partition the intensity along the boundaries of the field of view through recursive Gaussian splitting. However, this might still lead to a large number of components.

A different approach is proposed in [10], which introduces a version of the Gaussian mixture PHD filter where it is assumed that new targets are always detected. Thereby, the complicated representation of undetected targets is avoided. Furthermore, by allowing the target birth intensity to take on a uniform distribution for the part of the target state that is directly observed through the measurements, the need for a large Gaussian mixture representation of target birth is eliminated [10]. This approach can also be used in the PMBM filter but does not indicate whether undetected targets are more likely to be located in a particular region than elsewhere, which is the case when the sensor's field of view is smaller than the tracking region. It is thus not applicable in sensor management applications where the intensity of undetected targets is exploited when planning the search for new targets [8].

This paper builds upon the ideas of [10] and proposes a version of the PMBM filter where the birth intensity is gridbased, i.e., a mixture of disjoint weighted uniform distributions, for the part of the target state that is observed through the measurements, and Gaussian distributed for the unobserved state components. This allows the intensity of undetected targets to vary over the region of interest, potentially with sharp edges around the field of view. The structure of the intensity is preserved in the prediction step, which is computed using a simplified version of the Rao-Blackwellized point mass filter (RB-PMF) [11]. The grid-based representation allows the intensity of undetected targets to vary over the region of interest with sharp edges around the sensor's field 
of view, without using a large number of Gaussian mixture components. It should be noted that the complexity of the method depends on the number of grid points used, which thus is an important tuning parameter. Furthermore, it is shown that the density of potential targets detected for the first time can be approximated as Gaussian. This allows the prediction and measurement update of the MBM component to be performed as in a standard Gaussian PMBM filter implementation [6].

To highlight the potential benefits of the proposed filter, it is compared to a conventional PMBM filter with Gaussian mixture birth in a sensor management application, where a team of sensors are monitoring an area in order to search for and track an unknown number of targets.

The paper is organized as follows. Section II gives a brief introduction to RFS-based target tracking. The details of the proposed PMBM filter are presented in Section III, and in Section IV the filter is illustrated in a sensor management application. Conclusions are presented in Section V.

\section{BACKGROUND}

This section provides an overview of random finite set (RFS) models and multi-target filtering using RFS theory.

\section{A. Random finite set models}

A random finite set (RFS) is a set with a random number of elements which themselves are random [12]. This means that the cardinality of an RFS is a discrete random variable, and its elements are random variables. This makes the RFS framework convenient for representing sets of multiple targets and multiple sensor measurements. In this work, the following RFSs will be of interest:

1) Poisson point process: A Poisson point process (PPP) is an RFS of which the cardinality is Poisson distributed with rate $\mu$ and the elements, given the cardinality, are independent and identically distributed (IID) according to $p(x)$. The rate $\mu$ and distribution $p(x)$ form the intensity $\lambda(x)$ of the PPP as $\lambda(x)=\mu p(x)$. The density of a PPP $X$ is given by [13]:

$$
\pi(X)=e^{-\langle\lambda, 1\rangle} \prod_{x \in X} \lambda(x)
$$

where the notation $\langle a, b\rangle=\int a(x) b(x) \mathrm{d} x$ is used for the inner product of $a(x)$ and $b(x)$.

2) Bernoulli RFS: The cardinality of a Bernoulli RFS is Bernoulli distributed with parameter $r \in[0,1]$. It is either empty, with probability $1-r$, or, with probability $r$, contains a single element with density $p(x)$. Thus, the density of a Bernoulli RFS $X$ is [13]:

$$
\pi(X)= \begin{cases}1-r, & X=\emptyset \\ r p(x), & X=\{x\} \\ 0, & |X| \geq 2\end{cases}
$$

3) Multi-Bernoulli RFS: The disjoint union of a fixed number of Bernoulli RFSs is a multi-Bernoulli (MB) RFS. Its density is defined by the parameters $\left\{r^{i}, p^{i}\right\}_{i \in \mathbb{I}}$, where $\mathbb{I}$ is an index set:

$$
\pi(X)=\sum_{\uplus_{i} \in \mathbb{I} X^{i}=X} \prod_{i \in \mathbb{I}} \pi^{i}\left(X^{i}\right)
$$

The notation $X^{1} \uplus X^{2}=X$ denotes disjoint union, i.e., $X^{1} \cup X^{2}=X$ and $X^{1} \cap X^{2}=\emptyset$.

4) Multi-Bernoulli mixture RFS: A normalized, weighted sum of MB RFSs is referred to as a multi-Bernoulli mixture (MBM) RFS. Its density can be expressed as [14]:

$$
\pi(X)=\sum_{j \in \mathbb{J}} w^{j} \sum_{\uplus_{i \in \mathbb{I}^{j}} X^{i}=X} \prod_{i \in \mathbb{I}^{j}} \pi^{j, i}\left(X^{i}\right)
$$

and is defined by the set of parameters $\left\{w^{j},\left\{r^{j, i}, p^{j, i}\right\}_{i \in \mathbb{I}^{j}}\right\}_{j \in \mathbb{J}}$, where $\mathbb{J}$ is an index set for the MB components of the MBM, $\mathbb{I}^{j}$ is an index set for the Bernoullis of the $j$ th MB RFS, and $w^{j}$ is the weight of the $j$ th $\mathrm{MB}$ component.

\section{B. Multi-target filtering using RFS theory}

In the RFS-based filtering approach, the multi-target state and set of measurements at time $k$ are modeled as two RFSs denoted $X_{k}$ and $Z_{k}$, respectively. The aim is to estimate the posterior multi-target state density $\pi_{k \mid k}\left(X_{k} \mid Z_{1: k}\right)$, where $Z_{1: k}$ is a collection of finite sets of measurements received up to and including time $k$.

Similar to the standard single-target case, the multi-target posterior density can be computed recursively via prediction and measurement update steps. With the Bayes multi-target filter [12], the posterior multi-target density at time $k$ is propagated in time using the Chapman-Kolmogorov equation

$$
\pi_{k+1 \mid k}\left(X \mid Z_{1: k}\right)=\int \phi_{X}\left(X \mid X^{\prime}\right) \pi_{k \mid k}\left(X^{\prime} \mid Z_{1: k}\right) \delta X^{\prime},
$$

where $\phi_{X}\left(X \mid X^{\prime}\right)$ is the standard multi-target transition density, i.e., a Markovian process for individual targets with transition density $p_{k+1, k}\left(x \mid x^{\prime}\right)$ and state-dependent probability of survival $p_{\mathrm{s}, k}(x)$, combined with a PPP birth process with intensity $\lambda_{k}^{\mathrm{b}}(x)$.

Given a measurement set $Z_{k}$ with multi-target measurement set likelihood function $\phi_{Z}\left(Z_{k} \mid X_{k}\right)$, the predicted multi-target density is updated using Bayes' rule

$$
\pi_{k \mid k}\left(X \mid Z_{1: k}\right)=\frac{\phi_{Z}\left(Z_{k} \mid X\right) \pi_{k \mid k-1}\left(X \mid Z_{1: k-1}\right)}{\int \phi_{Z}\left(Z_{k} \mid X\right) \pi_{k \mid k-1}\left(X \mid Z_{1: k-1}\right) \delta X} .
$$

The standard multi-target measurement model [12] is used, which means that $\phi_{Z}(Z \mid X)$ models noisy measurements of individual targets with state-dependent probability of detection $p_{\mathrm{D}, k}(x)$, combined with PPP clutter with intensity $\lambda_{k}^{\mathrm{fa}}(z)$. At most one measurement is generated for each target in each time step, and each measurement is the result of at most one target. A measurement of a target is independent of all other targets and other measurements conditioned on the same target; the single target measurement likelihood is $p_{z_{k}}(x)=p\left(z_{k} \mid x\right)$. Note that the above functions in general depend on the sensor state, but this is implicit here for notational brevity. The integrals in (5) and (6) are set integrals, as defined in [12]. 


\section{PMBM FILTER WITH PARTIALLY UNIFORM TARGET BIRTH MODEL}

The PMBM filter estimates the state of the set of targets, i.e., the density $\pi$ of the RFS $X$, which is assumed to be a PMBM: a union of a PPP component (1) and an MBM component (4). The PPP represents targets that are hypothesized to exist, but have never been detected, e.g., targets that have been located in a region where the sensor system has low detection probability. The MBM represents targets that have been detected at least once, and each MB in the mixture corresponds to a unique sequence of data associations for all detected targets, referred to as a global hypothesis.

The general form of the PMBM filter recursion for the intensity of undetected targets $\lambda^{\mathrm{u}}(x)$ is given by

$$
\begin{aligned}
\lambda_{k+1 \mid k}^{\mathrm{u}}(x)= & \lambda_{k+1}^{\mathrm{b}}(x) \\
& +\int p_{\mathrm{s}, k}\left(x^{\prime}\right) p_{k+1, k}\left(x \mid x^{\prime}\right) \lambda_{k \mid k}^{\mathrm{u}}\left(x^{\prime}\right) \mathrm{d} x^{\prime}, \\
\lambda_{k \mid k}^{\mathrm{u}}(x)= & \left(1-p_{\mathrm{D}, k}(x)\right) \lambda_{k \mid k-1}^{\mathrm{u}}(x),
\end{aligned}
$$

where $\lambda_{k}^{\mathrm{b}}(x)$ is the intensity of target birth at time $k, p_{\mathrm{S}, k}(x)$ is the probability of survival for a target in state $x$ at time $k$, $p_{k+1, k}\left(x \mid x^{\prime}\right)$ is the target state transition density from time $k$ to time $k+1$, and $p_{\mathrm{D}, k}(x)$ is the probability of detection for a target in state $x$ at time $k$.

Each measurement $z_{k}$ at time $k$ generates a new potentially detected target, which is represented by a Bernoulli RFS with existence probability $r\left(z_{k}\right)$ and density $p\left(x \mid z_{k}\right)$ defined as

$$
\begin{aligned}
r\left(z_{k}\right) & =\frac{\int p_{\mathrm{D}, k}(x) p_{z_{k}}(x) \lambda_{k \mid k-1}^{\mathrm{u}}(x) \mathrm{d} x}{\lambda^{\mathrm{fa}}\left(z_{k}\right)+\int p_{\mathrm{D}, k}(x) p_{z_{k}}(x) \lambda_{k \mid k-1}^{\mathrm{u}}(x) \mathrm{d} x}, \\
p\left(x \mid z_{k}\right) & =\frac{p_{\mathrm{D}, k}(x) p_{z_{k}}(x) \lambda_{k \mid k-1}^{\mathrm{u}}(x)}{\int p_{\mathrm{D}, k}(x) p_{z_{k}}(x) \lambda_{k \mid k-1}^{\mathrm{u}}(x) \mathrm{d} x},
\end{aligned}
$$

where $p_{z_{k}}(x)=p\left(z_{k} \mid x\right)$ is the measurement likelihood function for a measurement $z_{k}$ given target state $x$.

In the following sections, approximations of (7) and (8) are derived for the case when the birth intensity is a union of a grid-based intensity and a Gaussian component. For the prediction and measurement update of existing tracks, the equations of the standard Gaussian implementation of the PMBM filter [6] can be used. The equations corresponding to these steps are outlined in Appendix A.

\section{A. Modeling}

Using the notation of [10], let $\theta$ represent the scalar or vector consisting of the part of the state $x$ that directly affects the measurement likelihood, i.e., $p_{z}(x)=p_{z}(\theta)$, and let $\varphi$ represent the remaining part of $x$. In the following, $x$ and $(\theta, \varphi)$ are used interchangeably. In the typical scenario with position measurements and state vector $x$ comprised of position and velocity, $\theta$ corresponds to the position components and $\varphi$ corresponds to the velocity components.

The intensity of undetected targets is modeled as the product of a grid-based intensity in $\theta$, i.e., a mixture of weighted disjoint uniform distributions, and a Gaussian distribution in $\varphi$. To facilitate the grid-based representation, the tracking volume in $\theta$ is partitioned into cells $\mathcal{C}^{(i)}, i=1, \ldots, N$. The cells are assumed to be mutually disjoint, i.e., $\mathcal{C}^{(i)} \cap \mathcal{C}^{(j)}=\emptyset$ for $i \neq j$, and the union of all cells $\bigcup_{i} \mathcal{C}^{(i)}$ covers the entire tracking volume in $\theta$. For simplicity, the cells are assumed to be axis aligned hyperrectangles. The midpoint and side lengths of cell $\mathcal{C}^{(i)}$ are denoted $\theta^{(i)}$ and $\Delta_{1}^{(i)}, \ldots, \Delta_{n_{\theta}}^{(i)}$, where $n_{\theta}=\operatorname{dim} \theta$. With $k^{\prime} \in\{k, k-1\}$, the intensity of undetected targets is given by

$$
\lambda_{k \mid k^{\prime}}^{\mathrm{u}}(\theta, \varphi)=\left[\sum_{i=1}^{N} w_{k \mid k^{\prime}}^{(i)} \mathcal{U}\left(\theta ; \mathcal{C}^{(i)}\right)\right] \mathcal{N}\left(\varphi ; \hat{\varphi}, P^{\varphi}\right)
$$

where $\mathcal{U}\left(\theta ; \mathcal{C}^{(i)}\right)$ is the uniform distribution in $\theta$ over the cell $\mathcal{C}^{(i)}, \hat{\varphi}$ is the prior mean of the unmeasured state component and $P^{\varphi}$ is its variance, and $w_{k \mid k^{\prime}}^{(i)}$ is the expected number of undetected targets with state component $\theta$ in cell $\mathcal{C}^{(i)}$. Note that a grid-based method implies a tradeoff between accuracy and complexity, and that grid-based methods suffer from the curse of dimensionality. However, as $\theta$ is often of low dimension (typically corresponding to the position components), the method is applicable in many tracking scenarios.

For the typical case where the target state consists of position and velocity, and the measurements correspond to the position, the model (9) corresponds to using a single prior distribution on the velocity in the entire region of interest and allowing the intensity of undetected targets be positiondependent.

It is assumed, as is commonly the case in tracking applications, that both the probability of survival $p_{\mathrm{s}, k}(x)$ and the probability of detection $p_{\mathrm{D}, k}(x)$ are independent of $\varphi$ and constant in each cell, i.e., $p_{\mathrm{S}, k}(x)=p_{\mathrm{S}, k}^{(i)}$ and $p_{\mathrm{D}, k}(x)=p_{\mathrm{D}, k}^{(i)}$ for all $x$ such that $\theta \in \mathcal{C}^{(i)}$. The measurement likelihood is assumed $\varphi$-independent and Gaussian distributed according to $p_{z}(x)=p_{z}(\theta)=\mathcal{N}\left(z ; h_{k}(\theta), R_{k}\right)$. Furthermore, let the components of $x$ be ordered such that the state transition function has the following structure:

$$
\underbrace{\left[\begin{array}{c}
\theta_{k+1} \\
\varphi_{k+1}
\end{array}\right]}_{x_{k+1}}=\underbrace{\left[\begin{array}{cc}
F_{k}^{\theta} & F_{k}^{\theta \varphi} \\
F_{k}^{\varphi \theta} & F_{k}^{\varphi}
\end{array}\right]}_{F_{k}} \underbrace{\left[\begin{array}{c}
\theta_{k} \\
\varphi_{k}
\end{array}\right]}_{x_{k}}+\underbrace{\left[\begin{array}{c}
w_{k}^{\theta} \\
w_{k}^{\varphi}
\end{array}\right]}_{w_{k}}
$$

where $p\left(w_{k}\right)=\mathcal{N}\left(w_{k} ; 0, Q_{k}\right)$.

\section{B. Prediction}

Let the posterior intensity of undetected targets at time $k$ be defined as in (9) with $k^{\prime}=k$ and define the birth intensity as

$$
\lambda_{k}^{\mathrm{b}}(\theta, \varphi)=\left[\sum_{i=1}^{N} w_{k}^{\mathrm{b}(i)} \mathcal{U}\left(\theta ; \mathcal{C}^{(i)}\right)\right] \mathcal{N}\left(\varphi ; \hat{\varphi}, P^{\varphi}\right),
$$

where $w_{k}^{\mathrm{b}(i)}$ is the expected number of targets with state component $\theta$ in cell $\mathcal{C}^{(i)}$ appearing at time $k$. The aim is to find an approximation of (7a) such that the predicted intensity $\lambda_{k+1 \mid k}^{\mathrm{u}}$ has the same form as (9). The birth intensity (11), which represents the first term in (7a), already has the same form as (9). This means that only the second term (the integral) 
in (7a) needs to be approximated such that it does not affect the intensity in $\varphi$ and maintains the grid-based intensity in $\theta$.

The RB-PMF [11] is used to approximate the predicted intensity. Given a posterior point-mass density

$$
\hat{p}_{k \mid k}(x)=\sum_{i=1}^{N} w_{k \mid k}^{(i)} \mathcal{U}\left(\theta ; \mathcal{C}^{(i)}\right) \mathcal{N}\left(\varphi ; \hat{\varphi}_{k \mid k}^{(i)}, P_{k \mid k}^{\varphi,(i)}\right),
$$

the RB-PMF prediction gives predicted weights $w_{k+1 \mid k}^{(j)}$ and a Gaussian distribution in $\varphi_{k+1}$ for each combination of $\theta_{k}^{(i)}$ and $\theta_{k+1}^{(j)}$. If the process noises acting on $\theta$ and $\varphi$ in (10) are independent, i.e., if the corresponding covariance matrix $Q_{k}$ is block-diagonal according to

$$
Q_{k}=\left[\begin{array}{cc}
Q_{k}^{\theta} & Q_{k}^{\theta \varphi} \\
\left(Q_{k}^{\theta \varphi}\right)^{\top} & Q_{k}^{\varphi}
\end{array}\right]=\left[\begin{array}{cc}
Q_{k}^{\theta} & 0 \\
0 & Q_{k}^{\varphi}
\end{array}\right]
$$

the predictive point-mass density is given by $[11,15]$

$$
\begin{aligned}
& \hat{p}_{k+1 \mid k}(x) \\
& \quad=\sum_{j=1}^{N} \sum_{i=1}^{N} w_{k+1 \mid k}^{(i, j)} \mathcal{U}\left(\theta ; \mathcal{C}^{(j)}\right) \mathcal{N}\left(\varphi ; \hat{\varphi}_{k+1 \mid k}^{(i, j)}, P_{k+1 \mid k}^{\varphi,(i, j)}\right),
\end{aligned}
$$

where

$$
\begin{aligned}
w_{k+1 \mid k}^{(i, j)} & =w_{k \mid k}^{(i)} \hat{\tilde{c}}^{(i, j)} \\
y_{k+1}^{(i, j)} & =\theta_{k+1}^{(j)}-F_{k}^{\theta} \theta_{k}^{(i)} \\
\hat{\tilde{c}}^{(i, j)} & =\mathcal{N}\left(y_{k+1}^{(i, j)} ; F_{k}^{\theta \varphi} \hat{\varphi}_{k \mid k}^{(i)}, F_{k}^{\theta \varphi} P_{k \mid k}^{\varphi,(i)}\left(F_{k}^{\theta \varphi}\right)^{\top}+Q_{k}^{\theta}\right)
\end{aligned}
$$

and

$$
\begin{aligned}
\hat{\varphi}_{k+1 \mid k}^{(i, j)} & =\mathbf{E}\left[\varphi_{k+1} \mid \theta_{k}=\theta^{(i)}, \theta_{k+1}=\theta^{(j)}, z_{1: k}\right] \\
& =F_{k}^{\varphi \theta} \theta^{(i)}+F_{k}^{\varphi} \hat{\varphi}_{k \mid k}^{(i, j)} \\
P_{k+1 \mid k}^{\varphi,(i, j)} & =\operatorname{cov}\left[\varphi_{k+1} \mid \theta_{k}=\theta^{(i)}, \theta_{k+1}=\theta^{(j)}, z_{1: k}\right] \\
& =F_{k}^{\varphi} P_{k \mid k}^{\varphi,(i, j)}\left(F_{k}^{\varphi}\right)^{\top}+Q_{k}^{\varphi} \\
\hat{\varphi}_{k \mid k}^{(i, j)} & =\hat{\varphi}_{k \mid k}^{(i)}+K_{k}^{(i, j)}\left(y_{k+1}^{(i, j)}-F_{k}^{\theta \varphi} \hat{\varphi}_{k \mid k}^{(i)}\right) \\
P_{k \mid k}^{\varphi,(i, j)} & =P_{k \mid k}^{\varphi,(i)}-K_{k}^{(i, j)} F_{k}^{\theta \varphi} P_{k \mid k}^{\varphi,(i)} \\
K_{k}^{(i, j)} & =P_{k \mid k}^{\varphi,(i)}\left(F_{k}^{\theta \varphi}\right)^{\top}\left(F_{k}^{\theta \varphi} P_{k \mid k}^{\varphi,(i)}\left(F_{k}^{\theta \varphi}\right)^{\top}+Q_{k}^{\theta}\right)^{-1}
\end{aligned}
$$

If the noise components are not independent, i.e., if $Q_{k}^{\theta \varphi} \neq 0$, the filter prediction can be modified to ensure independence of the noises affecting $\theta$ and $\varphi$ [16]. The reformulation exploits the property of the proposed method that $\theta_{k+1}^{(j)}$ is known when $\hat{\varphi}_{k+1 \mid k}^{(i, j)}$ and $P_{k \mid k}^{\varphi,(i, j)}$ are computed, see (16a) and (16b).

The increase in the number of Gaussian components means that the complexity of the RB-PMF is exponential, and the typical remedy to this is to merge the Gaussian mixture in $\varphi_{k+1}$ at each grid point $\theta_{k+1}^{(j)}$ using moment matching [11]. Here, the mixtures are instead replaced with the single Gaussian distribution $\mathcal{N}\left(\varphi ; \hat{\varphi}, P^{\varphi}\right)$. This means that no information about the state components $\varphi$ of undetected targets is inferred from the varying intensity in the state components $\theta$. Instead, the same prior on $\varphi$ is used in all grid points. As a consequence of the simplification, (16) does not need to be computed and the following approximation of the prediction step for the intensity of undetected targets (7a) is obtained:

$$
\lambda_{k+1 \mid k}^{\mathrm{u}}(\theta, \varphi) \approx\left[\sum_{j=1}^{N} w_{k+1 \mid k}^{(j)} \mathcal{U}\left(\theta ; \mathcal{C}^{(j)}\right)\right] \mathcal{N}\left(\varphi ; \hat{\varphi}, P^{\varphi}\right)
$$

where

$$
w_{k+1 \mid k}^{(j)}=w^{\mathrm{b}(j)}+\sum_{i=1}^{N} p_{\mathrm{s}, k}^{(i)} w_{k+1 \mid k}^{(i, j)}
$$

and $w_{k+1 \mid k}^{(i, j)}$ is given by (15) with $\hat{\varphi}_{k \mid k}^{(i)}=\hat{\varphi}$ and $P_{k \mid k}^{\varphi,(i)}=P^{\varphi}$. Note that (17) is in the same form as (7a) and that the prediction step computationally corresponds to a multi-dimensional convolution.

Remark 1: Note that if the computational complexity is not an issue, the standard RB-PMF approximation could be used to represent the intensity of undetected targets. However, as the complexity is quadratic in the number of grid points, this approach quickly becomes computationally expensive for anything but small examples as the entire tracking volume must be covered by the grid.

Remark 2: The approach can also be extended to handle birth intensities with several Gaussian components in $\varphi$, each with an associated grid-based intensity in $\theta$. For the typical case where the target state consists of position and velocity and the measurements correspond to the position, this could be used to model that targets are expected to appear with different velocities in different regions of the tracking volume.

\section{Update}

Let the predicted intensity of undetected targets at time $k$ be in the form (9), with $k^{\prime}=k-1$.

1) Intensity of undetected targets: The updated intensity of undetected targets (7b) is straightforward to compute as

$$
\lambda_{k \mid k}^{\mathrm{u}}(\theta, \varphi)=\sum_{i=1}^{N} w_{k \mid k}^{(i)} \mathcal{U}\left(\theta ; \mathcal{C}^{(i)}\right) \mathcal{N}\left(\varphi ; \hat{\varphi}, P^{\varphi}\right),
$$

where $w_{k \mid k}^{(i)}=\left(1-p_{\mathrm{D}, k}^{(i)}\right) w_{k \mid k-1}^{(i)}$.

2) Potential targets detected for the first time: The aim here is to find an approximation of (8) such that $p\left(x \mid z_{k}\right)$ is a Gaussian distribution. An approach consisting of two steps is employed for this. First, a point-mass-based approximation of the posterior density $(8 \mathrm{~b})$ is computed by performing the RBPMF measurement update step. The first two moments of this density are then computed to obtain a Gaussian approximation.

Given a predictive density

$$
\hat{p}_{k \mid k-1}(x)=\sum_{i=1}^{N} w_{k \mid k-1}^{(i)} \mathcal{U}\left(\theta ; \mathcal{C}^{(i)}\right) \mathcal{N}\left(\varphi ; \hat{\varphi}_{k \mid k-1}^{(i)}, P_{k \mid k-1}^{\varphi,(i)}\right),
$$

and a Gaussian measurement likelihood function $p_{z}(\theta)=$ $\mathcal{N}\left(z ; h_{k}(\theta), R_{k}\right)$ that is independent of $\varphi$, the RB-PMF measurement update step results in $[11,15]$

$$
\hat{p}_{k \mid k}(\theta)=\sum_{i=1}^{N} w_{k \mid k}^{(i)} \mathcal{U}\left(\theta ; \mathcal{C}^{(i)}\right) \mathcal{N}\left(\varphi ; \hat{\varphi}_{k \mid k}^{(i)}, P_{k \mid k}^{\varphi,(i)}\right),
$$


where

$$
\begin{aligned}
w_{k \mid k}^{(i)} & =\hat{\tilde{c}}_{k}^{-1} p_{z_{k}}^{(i)} w_{k \mid k-1}^{(i)} \\
\hat{\tilde{c}}_{k} & =\sum_{i=1}^{N} p_{z_{k}}^{(i)} w_{k \mid k-1}^{(i)} \\
\hat{\varphi}_{k \mid k}^{(i)} & =\hat{\varphi}_{k \mid k-1}^{(i)} \\
P_{k \mid k}^{\varphi((i)} & =P_{k \mid k-1}^{\varphi,(i)},
\end{aligned}
$$

where $p_{z_{k}}^{(i)}=\mathcal{N}\left(z_{k} ; h_{k}\left(\theta^{(i)}\right), R_{k}\right)$ is the measurement likelihood evaluated at the $i$ th grid point. As the measurement likelihood function is assumed to be independent of $\varphi$, the density remains unchanged with respect to these state components.

Using (21) and (22), a point-mass-based approximation of (8b) can be obtained as

$$
\hat{p}\left(x \mid z_{k}\right)=\sum_{i=1}^{N} w_{k \mid k}^{(i)}\left(z_{k}\right) \mathcal{U}\left(\theta ; \mathcal{C}^{(i)}\right) \mathcal{N}\left(\varphi ; \hat{\varphi}, P^{\varphi}\right),
$$

where

$$
\begin{aligned}
w_{k \mid k}^{(i)}\left(z_{k}\right) & =\hat{\tilde{c}}_{z_{k}}^{-1} p_{\mathrm{D}, k}^{(i)} p_{z_{k}}^{(i)} w_{k \mid k-1}^{(i)} \\
\hat{\tilde{c}}_{z_{k}} & =\sum_{i=1}^{N} p_{\mathrm{D}, k}^{(i)} p_{z_{k}}^{(i)} w_{k \mid k-1}^{(i)} .
\end{aligned}
$$

The first two moments of the point-mass density of $\theta$, i.e.,

$$
\hat{p}\left(\theta \mid z_{k}\right)=\sum_{i=1}^{N} w_{k \mid k}^{(i)}\left(z_{k}\right) \mathcal{U}\left(\theta ; \mathcal{C}^{(i)}\right)
$$

are given as

$$
\begin{aligned}
\hat{\theta}\left(z_{k}\right) & =\sum_{i=1}^{N} w_{k \mid k}^{(i)}\left(z_{k}\right) \theta^{(i)} \\
P^{\theta}\left(z_{k}\right) & =\sum_{i=1}^{N} w_{k \mid k}^{(i)}\left(z_{k}\right)\left[\left(\theta^{(i)}-\hat{\theta}\left(z_{k}\right)\right)\left(\theta^{(i)}-\hat{\theta}\left(z_{k}\right)\right)^{\top}+\Sigma_{\mathcal{U}}^{(i)}\right]
\end{aligned}
$$

where $\Sigma_{\mathcal{U}}^{(i)}=\operatorname{diag}\left(\left[\left(\Delta_{1}^{(i)}\right)^{2}, \ldots,\left(\Delta_{n \theta}^{(i)}\right)^{2}\right] / 12\right)$ is the covariance matrix of the uniform distribution over cell $\mathcal{C}^{(i)}$.

A Gaussian approximation of (23) is obtained by matching the moments using (26). Furthermore, note that $\hat{\tilde{c}}_{z_{k}}$ in (24b) corresponds to the denominator of (8b). The desired approximation of (8) is thus given by

$$
\begin{aligned}
r\left(z_{k}\right) & \approx \frac{\hat{\tilde{c}}_{z_{k}}}{\lambda_{k}^{\mathrm{fa}}\left(z_{k}\right)+\hat{\tilde{c}}_{z_{k}}}, \\
p\left(x \mid z_{k}\right) & \approx \mathcal{N}\left(\theta ; \hat{\theta}\left(z_{k}\right), P^{\theta}\left(z_{k}\right)\right) \mathcal{N}\left(\varphi ; \hat{\varphi}, P^{\varphi}\right) .
\end{aligned}
$$

\section{APPLiCATION TO SENSOR MANAGEMENT}

In this section, the proposed version of the PMBM filter is used as an underlying estimator in a multi-target sensor management problem. In the considered scenario, a team of controllable sensors are used to search for and estimate the states of an unknown number of targets. As the PMBM filter not only provides estimated states of the discovered targets, but also a representation of where previously undetected targets are likely to be found, it provides an appealing foundation for a unified search and track method [8]. Based on the PMBM density, the sensors' control inputs can be optimized in order to simultaneously maintain track of discovered targets and search for targets that are yet to be detected.

\section{A. Problem formulation}

At time $k$, the problem of computing the control inputs for a team of $S$ sensors for time steps $k$ up to $k+T-1$ is formulated as the following stochastic optimal control problem,

$$
\begin{array}{ll}
\underset{u_{k: k+T-1}^{1: S}}{\operatorname{minimize}} & \mathbf{E}\left[\sum_{t=k+1}^{k+T} \ell\left(\pi_{t \mid t}\right)\right] \\
\text { subject to } & u_{k: k+T-1}^{m} \in \mathcal{P}_{k}^{m}, \\
& s_{t}^{m}=g_{t}\left(s_{k}^{m}, u_{k: t}^{m}\right), \\
& \pi_{t+1 \mid t+1}=\rho\left(\pi_{t \mid t},\left\{\hat{Z}_{t+1}^{m}, s_{t+1}^{m}\right\}_{m=1}^{S}\right),
\end{array}
$$

where the expectation is taken with respect to the future measurement sets and

- $\pi_{t \mid t}$ is the posterior PMBM density at time $t$,

- $T$ is the planning horizon,

- $u_{k: k+T-1}^{m}$ is a sequence of control inputs from times $k$ up to $k+T-1$ for sensor $m$,

- $u_{k: k+T-1}^{1: S}$ is a collection of sequences of control inputs for $S$ sensors,

- $\mathcal{P}_{k}^{m}$ is a discrete set of all admissible sequences of control inputs at time $k$ for sensor $m$,

- $g_{t}\left(s_{k}^{m}, u_{k: t}^{m}\right)$ is a function that returns the sensor state $s_{t}^{m}$ at time $t$ if the control sequence $u_{k: t}^{m}$ is applied to sensor $m$ with state $s_{k}^{m}$ at time $k$,

- $\hat{Z}_{t}^{m}$ is a hypothesized set of measurements obtained by sensor $m$ at time $t$,

- $\rho\left(\pi_{t \mid t},\left\{\hat{Z}_{t+1}^{m}, s_{t+1}^{m}\right\}_{m=1}^{S}\right)$ is a shorthand notation for a prediction step followed by a sequence of update steps (one for each sensor $m$ ), and

- $\ell(\cdot)$ is a stage cost function used to trade-off between tracking discovered targets and searching for new targets.

Since the targets maneuver and the scenario changes over time, it is necessary to re-plan the sequences of sensor control inputs online as new measurements are obtained. This is done in standard receding horizon fashion [17], i.e., for each sensor, only the first element of the computed sequence of control inputs is applied before re-planning.

1) Predicted measurement sets: The computationally expensive expectation over the future measurement sets in (28) is avoided using the predicted ideal measurement set approach [18]. This corresponds to assuming that the measurement sets $\hat{Z}_{t}^{m}$, for $t=k+1, \ldots, k+T$ and $m=1, \ldots, S$, are generated without misdetections, measurement noise, and false alarms. For each sensor and time step, a noise-free measurement is generated for each target that is predicted to be within field of view. Let $\hat{X}_{t \mid k}$ denote a set of predicted target states at time $t$. The predicted ideal measurement set $\hat{Z}_{t}^{m}$ corresponding to a sensor with state $s_{t}^{m}$ and field of view $\mathcal{V}\left(s_{t}^{m}\right)$ is then given by

$$
\hat{Z}_{t}^{m}=\bigcup_{\hat{x} \in \hat{X}_{t \mid k}: \hat{x} \in \mathcal{V}\left(s_{t}^{m}\right)}\left\{\underset{z}{\arg \max } p\left(z \mid \hat{x}, s_{t}^{m}\right)\right\},
$$


where $p(z \mid x, s)$ is the single target measurement likelihood function.

The set of predicted target states $\hat{X}_{t \mid k}$ is extracted from the hypothesis with highest weight in the MBM component of the predicted PMBM density $\pi_{t \mid k}$. The index of the MB corresponding to this hypothesis is denoted $j^{\star}$ and obtained as

$$
j^{\star}=\underset{j \in \mathbb{J}}{\arg \max } w^{j},
$$

where $w^{j}$ and $\mathbb{I}$ are parameters of the MBM density (4). The Bernoulli components in this MB with probability of existence at time $k$ greater than a threshold $\Gamma$ are selected, and the expected values of the corresponding densities are included in the set of predicted target states. Assuming that the predicted single-target state densities are Gaussian, i.e., $p_{t \mid k}^{j^{\star}, i}(x)=\mathcal{N}\left(x ; \hat{x}_{t \mid k}^{j^{\star}, i}, P_{t \mid k}^{j^{\star}, i}\right)$ for $i \in \mathbb{I}^{j^{\star}}$, the set is given as

$$
\hat{X}_{t \mid k}=\left\{\hat{x}_{t \mid k}^{j^{\star}, i}\right\}_{i \in \mathbb{I}^{j}: r_{k \mid k}^{j^{\star}, i}>\Gamma^{*}}
$$

2) Objective function: The objective function proposed in [8] is employed, i.e., the cost function $\ell\left(\pi_{t \mid t}\right)$ is a weighted sum of two terms,

$$
\ell\left(\pi_{t \mid t}\right)=\ell^{\text {track }}\left(\pi_{t \mid t}\right)+\eta \ell^{\text {search }}\left(\pi_{t \mid t}\right),
$$

where $\ell^{\text {track }}$ captures the tracking performance, $\ell^{\text {search }}$ captures the search performance, and $\eta$ is a user-defined non-negative weight used to trade-off between tracking and searching [8].

The tracking performance is evaluated based on the uncertainty in the resulting target state estimates. The uncertainty is quantified by summing the trace of the covariance matrix of each track:

$$
\ell^{\text {track }}\left(\pi_{t \mid t}\right)=\sum_{i \in \mathbb{I}^{\star}} \operatorname{tr}\left(W P_{t \mid t}^{j^{\star}, i} W^{\top}\right),
$$

where $W$ is a positive semidefinite matrix of appropriate dimensions used to extract and weigh the relevant components of the covariance matrix. The variable $P_{t \mid t}^{j^{\star}, i}$ is the covariance matrix of track $i$ in hypothesis $j^{\star}$ at time $t$ given the actual measurements up until time $k$ and predicted ideal measurements from time $k+1$ to $t$.

The search performance is captured by the expected number of undetected targets. This is given by the integral of the intensity of undetected targets, which is equal to the sum of the weights in point mass intensity:

$$
\ell^{\text {search }}\left(\pi_{t \mid t}\right)=\int \lambda_{t \mid t}^{\mathrm{u}}(x) \mathrm{d} x=\sum_{i=1}^{N} w_{t \mid t}^{(i)} .
$$

Here, $\lambda_{t \mid t}^{\mathrm{u}}$ is the resulting intensity of undetected targets at time $t$ given the actual sensor trajectories up until time $k$ and planned trajectories from time $k+1$ to $t$. With this choice of cost functions, the parameter $\eta$ can be considered as the cost related to failing to discover an existing target [8].

3) Greedy assignment: The optimization problem in (28) scales exponentially with the number of sensors. Instead of solving the problem jointly for all sensors, the sequentially greedy approximation strategy of [19] is utilized to assign control sequences to each sensor. Initially, each sensor computes the objective function value for each control sequence

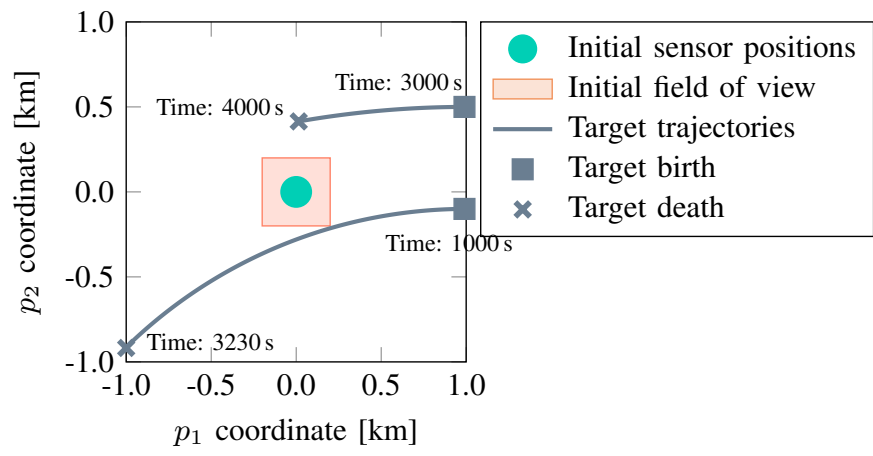

Fig. 1. Target trajectories and initial sensor states in the considered scenario.

$u_{k: k+T-1}^{m} \in \mathcal{P}_{k}^{m}$, without considering the other sensors. The combination of sensor and control sequence with the smallest objective function value is selected. The remainder of the team then recomputes the objective function value of each control sequence conditioned on the selected control sequence of the first sensor, and the sensor and control sequence with the lowest cost are again selected. This process repeats until all sensors have been assigned a control sequence.

\section{B. Simulation results}

The simulated scenario involves a team of two sensors with limited fields of view that are tracking an unknown and timevarying number of targets. Both sensors are initially located at the origin. No targets are present at the beginning of the considered scenario, with one arriving at time $1000 \mathrm{~s}$, and another one at time $3000 \mathrm{~s}$. The first target leaves the scene at $3230 \mathrm{~s}$ and the second one remains until the end of the scenario, which lasts for $4000 \mathrm{~s}$. Fig. 1 illustrates the scenario geometry.

The single-target state $x=\left[p_{1}, v_{1}, p_{2}, v_{2}\right]^{\top}$ consists of its two-dimensional position and velocity and its dynamics follow a nearly constant velocity model, i.e., $p_{k+1, k}\left(x \mid x^{\prime}\right)=$ $\mathcal{N}\left(x ; F_{\mathrm{CV}} x^{\prime}, Q\right)$, where

$$
F_{\mathrm{CV}}=I_{2} \otimes\left[\begin{array}{ll}
1 & \tau \\
0 & 1
\end{array}\right], \quad Q=\sigma_{w}^{2} G G^{\top}, \quad G=I_{2} \otimes\left[\begin{array}{c}
\frac{\tau^{2}}{2} \\
\tau
\end{array}\right],
$$

where $\otimes$ is the Kronecker product, $\tau=10 \mathrm{~s}$ is the sampling period, and $\sigma_{w}=0.05 \mathrm{~m} / \mathrm{s}^{2}$ is the standard deviation for the target acceleration. The survival probability is assumed constant $p_{\mathrm{S}}=0.99$ for each target.

A sensor with state $s$ at position $\left(p_{1}^{\mathrm{s}}, p_{2}^{\mathrm{s}}\right)$ has field of view $\mathcal{V}(s)$ in the form of a square with sides $a=400 \mathrm{~m}$ centered around its position,

$$
\mathcal{V}(s)=\left\{x: \max \left(\left|p_{1}-p_{1}^{s}\right|,\left|p_{2}-p_{2}^{s}\right|\right) \leq a / 2\right\}
$$

The detection probability is constant within the field of view and each detection results in a linear measurement of the position of the corresponding target, i.e.,

$$
\begin{aligned}
p_{\mathrm{D}}(x \mid s) & = \begin{cases}0.9, & \text { if } x \in \mathcal{V}(s), \\
0, & \text { otherwise. }\end{cases} \\
p(z \mid x, s) & = \begin{cases}\mathcal{N}(z ; H x, R), & \text { if } x \in \mathcal{V}(s), \\
0, & \text { otherwise. }\end{cases}
\end{aligned}
$$


with parameters

$$
H=I_{2} \otimes\left[\begin{array}{ll}
1 & 0
\end{array}\right], \quad R=\sigma_{p}^{2} I_{2} .
$$

where $\sigma_{p}=10 \mathrm{~m}$ is the standard deviation of the position measurement noise. The clutter is modeled by a Poisson RFS with uniform intensity in the field of view and five expected false alarms per time step.

The part of the target state $x$ that is involved in the measurement $z$ corresponds to the position components, i.e., $\theta=\left[p_{1}, p_{2}\right]^{\top}$ and $\varphi=\left[v_{1}, v_{2}\right]^{\top}$. To model the intensity of undetected targets, a grid of size $201 \times 201$ over $\theta$ that covers the surveillance region is defined. This corresponds to grid cells of size $10 \mathrm{~m} \times 10 \mathrm{~m}$ centered around the points $\theta^{(i)}=\left[p_{1}^{(i)}, p_{2}^{(i)}\right]^{\top}$. The birth intensity is defined as in (11) with

$$
\begin{aligned}
w_{k}^{\mathrm{b}(i)} & = \begin{cases}0.01 / 201, & i: p_{1}^{(i)}=1000, \\
0, & \text { otherwise, }\end{cases} \\
\hat{\varphi} & =[-1,0]^{\top}, \\
P^{\varphi} & =I_{2} .
\end{aligned}
$$

i.e., at each time step 0.01 new targets are expected to arrive from the right and travel towards the left with $1 \mathrm{~m} / \mathrm{s}$.

The state of each mobile sensor $s=\left[p_{1}^{\mathrm{s}}, p_{2}^{\mathrm{s}}, \alpha\right]^{\top}$ consists of its two-dimensional position and heading. The sensors move according to discretized differential drive dynamics

$$
\left[\begin{array}{c}
p_{1, k+1}^{\mathrm{s}} \\
p_{2, k+1}^{\mathrm{s}} \\
\alpha_{k+1}
\end{array}\right]=\left[\begin{array}{c}
p_{1, k}^{\mathrm{s}} \\
p_{2, k}^{\mathrm{s}} \\
\alpha_{k}
\end{array}\right]+\left[\begin{array}{c}
\tau \nu \operatorname{sinc}\left(\frac{\tau \omega}{2}\right) \cos \left(\alpha_{k}+\frac{\tau \omega}{2}\right) \\
\tau \nu \operatorname{sinc}\left(\frac{\tau \omega}{2}\right) \sin \left(\alpha_{k}+\frac{\tau \omega}{2}\right) \\
\tau \omega
\end{array}\right]
$$

with constant speed of $\nu=5 \mathrm{~m} / \mathrm{s}$ and heading rate $\omega$ determined by the control input. Each admissible sequence of control inputs corresponds to a heading change followed by a straight path. The heading changes are performed at a constant turn rate of $|\omega|=\pi / 10 \mathrm{rad} / \mathrm{s}$ and the set of allowed heading changes is $\{n \pi / 6 \mathrm{rad}, n \in\{-6,-5, \ldots, 6\}\}$. The duration of each maneuver is $150 \mathrm{~s}$, which corresponds to a planning horizon of $T=15$ measurements.

Fig. 2 shows snapshots from one realization of the scenario. New targets are expected to arrive from the right hand side, and the light blue areas illustrate the intensity of undetected targets. During the first $1000 \mathrm{~s}$ of the simulation, when no targets are present, the two sensors patrol the right edge of the surveillance area in order to detect any appearing targets. When the first target appears, the two sensors split up. One of the sensors tracks the target and the other one continues to search for new targets along the edge of the area. As the second target arrives at time $3000 \mathrm{~s}$, the sensor that was patrolling the edge of the area has to track the new target while searching for undetected targets nearby. The first sensor continues to track the first target until it leaves the area, after which the sensor returns to the right edge of the area to search for new targets. The sensors' behavior indicates that the planning algorithm works as intended: the team utilizes the available resources to concurrently search for new targets and track the targets that have been detected.

To evaluate the tracking performance of the PMBM filter with the proposed grid-based birth intensity, it is compared to a conventional PMBM filter that uses a standard Gaussian mixture birth intensity. The Gaussian mixture birth intensity is designed to approximate the grid-based birth intensity using a reasonable number of components. It is given by $\lambda^{\mathrm{b}}(x)=\sum_{i=1}^{N^{\mathrm{b}}} w^{\mathrm{b}, i} \mathcal{N}\left(x ; \hat{x}^{\mathrm{b}, i}, P^{\mathrm{b}, i}\right)$, with

$$
\begin{aligned}
N^{\mathrm{b}} & =9, \\
w^{\mathrm{b}, i} & =0.01 / N^{\mathrm{b}}, \\
P^{\mathrm{b}, i} & =\operatorname{diag}\left(\left[5^{2}, 1,125^{2}, 1\right]\right), \\
\hat{x}^{\mathrm{b}, i} & =\left[1000,-1, \hat{p}_{2}^{\mathrm{b}, i}, 0\right]^{\top},
\end{aligned}
$$

where $\hat{p}_{2}^{\mathrm{b}, i}=-1000+250(i-1)$ for $i=1, \ldots, N^{\mathrm{b}}$. The same scenario and planning algorithm parameters are used for both methods, the only difference is the underlying representation of the intensity of undetected targets. To simplify the computations, the probability of detection is approximated as constant for each Gaussian component, determined by the probability of detection at its expected value.

The generalized optimal subpattern assignment (GOSPA) metric [20] is used to evaluate the performance of both methods. It is a unified performance metric for multi-target tracking that penalizes both localization errors for detected targets and errors due to missed and false targets. Fig. 3 shows the GOSPA metric (with parameters $\alpha=2$, order two, localization error $d(x, y)$ defined as the 2-norm of the position components of $x-y$, and maximum allowable localization error $50 \mathrm{~m}$ ) averaged over 100 Monte Carlo runs. The peaks in the GOSPA metric at $1000 \mathrm{~s}$ and $3000 \mathrm{~s}$ occur when new targets appear. The performance in terms of GOSPA is similar for both birth models. Fig. 3 also shows the average number of missed and false targets during the scenario. There is no significant difference in the number of false targets, but the sensors detect the targets more often, i.e., miss fewer targets, when the intensity of undetected targets is modeled using the grid-based model compared to when it is modeled using the Gaussian mixture.

As a second performance measure, the computation time needed for a full filter iteration, i.e., a prediction step and a measurement update step, is analyzed. Although this comparison is based on non-optimized Matlab implementations, it gives an indication of the methods' relative computational complexity. For more insight, the computation time is split into two parts: (i) time needed for maintaining existing tracks, and (ii) time needed for handling the intensity of undetected targets and initiation of new tracks. The computation times at each time step of the scenario averaged over 100 Monte Carlo simulations are shown in Fig. 4. The proposed method leads to slightly more time being spent on track maintenance. This is as expected, as fewer targets are missed with this method and the equations that correspond to track maintenance are the same regardless of the choice of birth model, see Appendix A. The computational benefit of the proposed method in this scenario is a reduction in the time needed for handling the intensity of undetected targets and initiation of new tracks. For the partially grid-based birth model, the time spent on this step is fairly constant throughout the scenario and significantly less than the time spent on maintaining existing tracks. This is in contrast to the Gaussian mixture birth model, where the time needed 
Time: $1000 \mathrm{~s}$

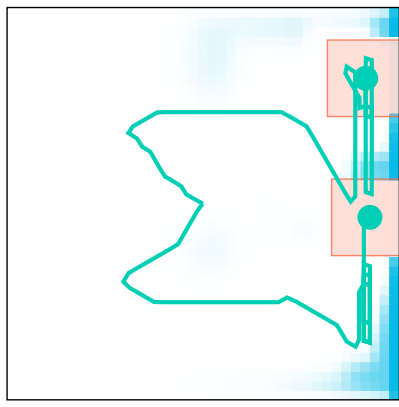

Time: $2500 \mathrm{~s}$

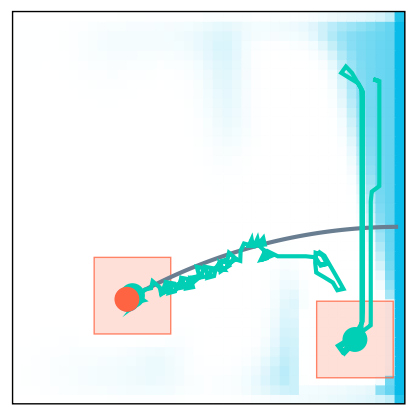

Time: $3500 \mathrm{~s}$

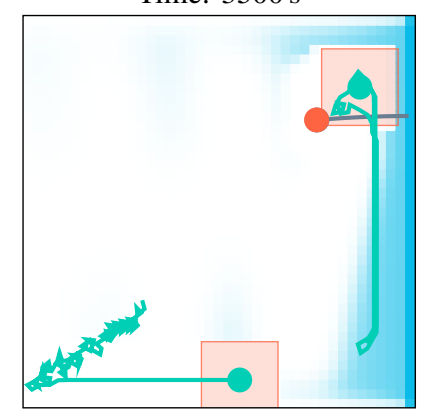

Time: $4000 \mathrm{~s}$

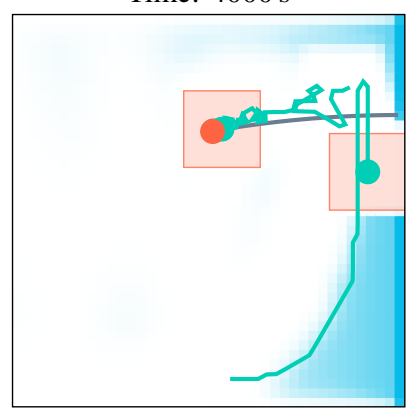

\begin{tabular}{|lll|}
\hline Sensor positions & Estimated target positions & Field of view \\
Sensor trajectories & True target trajectories & Intensity of undetected targets \\
\hline
\end{tabular}

Fig. 2. Snapshots of the simulated scenario where the sensor trajectories are planned for joint search and track based on the estimated PMBM density. The proposed representation of intensity of undetected targets is illustrated in white-blue scale, with a deeper blue indicating a higher intensity.
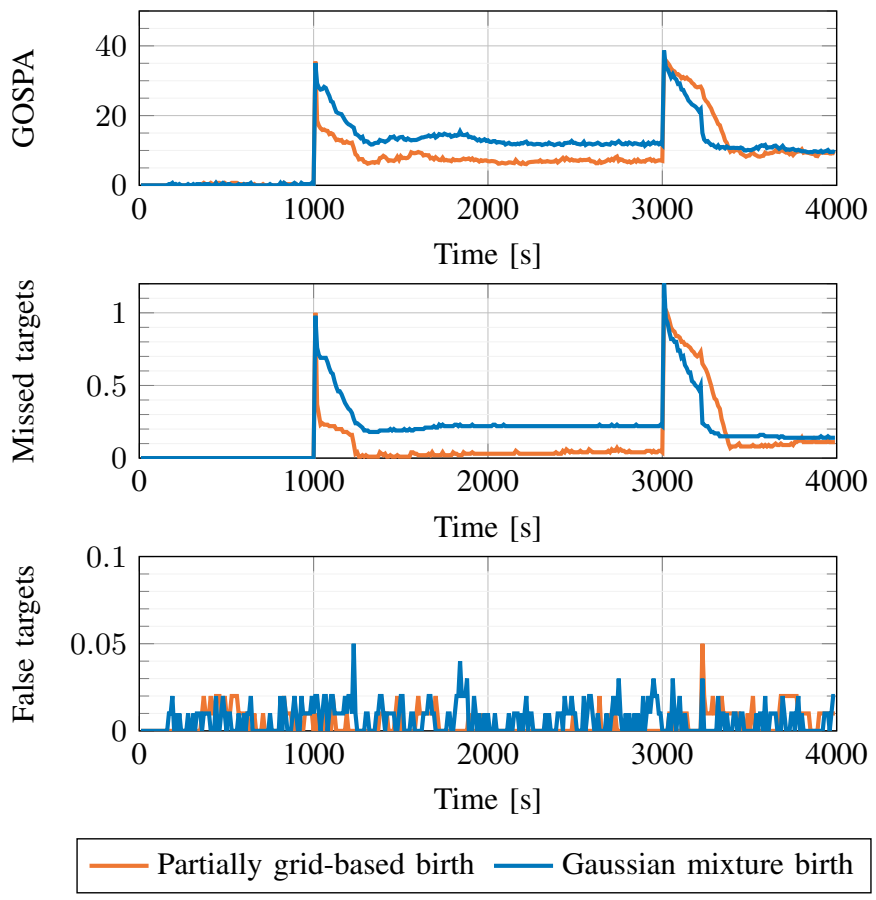

Fig. 3. Comparison of performance measures (averaged over 100 Monte Carlo simulations) for the proposed grid-based birth model and the conventional Gaussian mixture birth model.

for this step depends on the number of components in the Gaussian mixture, which varies over time. As a large number of components are needed, significantly more time is spent on handling the intensity of undetected targets and initiation of new tracks than on maintaining existing tracks.

\section{CONCLUSIONS}

A PMBM filter with a new method to represent where yet undetected targets may be located has been derived. It relies on a grid-based intensity of undetected targets, which is useful in scenarios where the sensor's field of view does not cover the
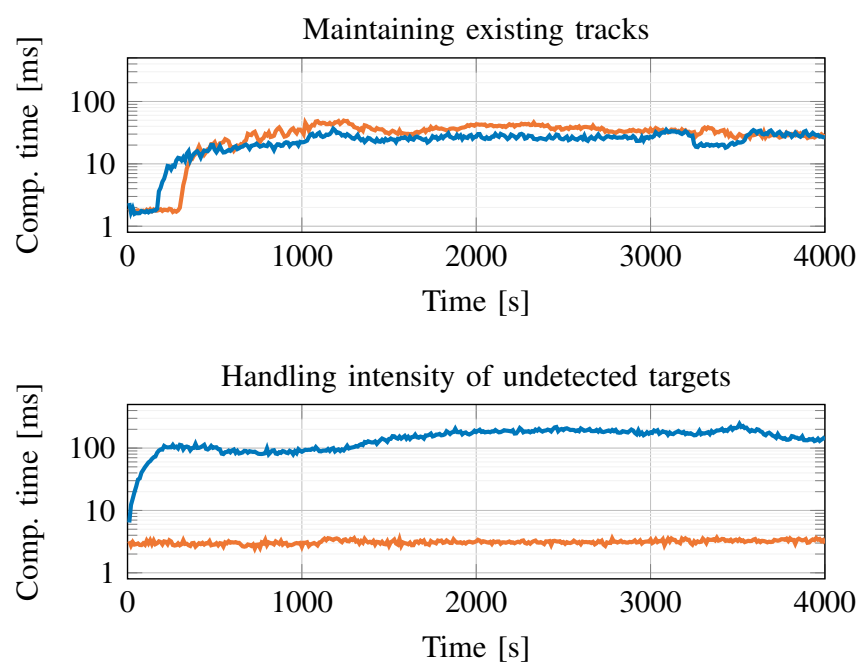

_ Partially grid-based birth _ Gaussian mixture birth

Fig. 4. Computation time needed for the PMBM filter with the proposed gridbased birth model and with the conventional Gaussian mixture birth model.

entire region of interest, as it allows for efficient representation of abrupt changes in the distribution. The intensity of undetected targets is estimated using Rao-Blackwellization, and the computational complexity of the prediction step is reduced using an approximation of an intermediate distribution. The overall computational complexity of the methods depends on the number of grid points. However, a simulation study showed that the proposed method operates at significantly lower computational cost and provides similar tracking performance as the conventional filter. The reduced computational complexity makes the proposed method suitable for sensor management applications, as these require methods that are both accurate and fast. 


\section{ACKNOWLEDGMENTS}

The authors would like to acknowledge the contribution by the anonymous reviewer who suggested the approach to initialize new tracks by exploiting the point mass approximation. The suggestion provided new insights, generalized the method, and resulted in a better approximation than the original approach.

\section{REFERENCES}

[1] R. Mahler, Advances in statistical Multisource-Multitarget Information Fusion. Norwood, MA, USA: Artech House, 2014.

[2] —_, "Multitarget Bayes filtering via first-order multitarget moments," IEEE Trans. Aerosp. Electron. Syst., vol. 39, no. 4, pp. 1152-1178, 2003

[3] B.-T. Vo and B.-N. Vo, "Labeled random finite sets and multi-object conjugate priors," IEEE Transactions on Signal Processing, vol. 61, no. 13 , pp. 3460-3475, 2013.

[4] B.-N. Vo, B.-T. Vo, and D. Phung, "Labeled random finite sets and the Bayes multi-target tracking filter,' IEEE Trans. Signal Process., vol. 62, no. 24, pp. 6554-6567, 2014.

[5] J. L. Williams, "Marginal multi-Bernoulli filters: RFS derivation of MHT, JIPDA, and association-based MeMBer," IEEE Trans. Aerosp. Electron. Syst., vol. 51, no. 3, pp. 1664-1687, 2015.

[6] Á. F. García-Fernández, J. L. Williams, K. Granström, and L. Svensson, "Poisson multi-Bernoulli mixture filter: direct derivation and implementation," IEEE Trans. Aerosp. Electron. Syst., vol. 54, no. 4, pp. 1883 1901, 2018.

[7] M. Fröhle, C. Lindberg, K. Granström, and H. Wymeersch, "Multisensor Poisson multi-Bernoulli filter for joint target-sensor state tracking," IEEE Trans. Intell. Veh., vol. 4, no. 4, pp. 609-621, 2019.

[8] P. Boström-Rost, D. Axehill, and G. Hendeby, "Sensor management for search and track using the Poisson multi-Bernoulli mixture filter," IEEE Trans. Aerosp. Electron. Syst., 2021, doi:10.1109/TAES.2021.3061802.

[9] K. LeGrand and S. Ferrari, "The role of bounded fields-of-view and negative information in finite set statistics (FISST)," in Proceedings of the 23rd International Conference on Information Fusion, 2020, pp. 1-9.

[10] M. Beard, B.-T. Vo, B.-N. Vo, and S. Arulampalam, "A partially uniform target birth model for Gaussian mixture PHD/CPHD filtering," IEEE Trans. Aerosp. Electron. Syst., vol. 49, no. 4, pp. 2835-2844, 2013.

[11] V. Šmídl and M. Gašperin, "Rao-Blackwellized point mass filter for reliable state estimation," in Proceedings of the 16th International Conference on Information Fusion, Istanbul, Turkey, 2013, pp. 312-318.

[12] R. Mahler, Statistical Multisource-Multitarget Information Fusion. Norwood, MA, USA: Artech House, 2007.

[13] B. Ristic, B.-T. Vo, B.-N. Vo, and A. Farina, "A tutorial on Bernoulli filters: theory, implementation and applications," IEEE Trans. Signal Process., vol. 61, no. 13, pp. 3406-3430, 2013.

[14] K. Granström, M. Fatemi, and L. Svensson, "Poisson multi-Bernoulli mixture conjugate prior for multiple extended target filtering," IEEE Trans. Aerosp. Electron. Syst., vol. 56, no. 1, pp. 208-225, 2020.

[15] J. Duník and O. Straka, "Design of Rao-Blackwellized point-mass smoother for conditionally linear and Gaussian models," IEEE Trans. Signal Process., vol. 67, no. 23, pp. 6053-6066, 2019.

[16] T. Schön, F. Gustafsson, and P.-J. Nordlund, "Marginalized particle filters for mixed linear/nonlinear state-space models," IEEE Trans. Signal Process., vol. 53, no. 7, pp. 2279-2289, 2005.

[17] J. M. Maciejowski, Predictive control: With constraints. Englewood Cliffs, NJ, USA: Prentice Hall, 2002.

[18] R. Mahler, "Multitarget sensor management of dispersed mobile sensors," in Theory and Algorithms for Cooperative Systems, D. Grundel, R. Murphey, and P. Pardalos, Eds. Singapore: World Scientific Publishing Co, 2004, ch. 12, pp. 239-310.

[19] P. M. Dames and V. Kumar, "Autonomous localization of an unknown number of targets without data association using teams of mobile sensors," IEEE Trans. Autom. Sci. Eng., vol. 12, no. 3, pp. 850-864, 2015.

[20] A. S. Rahmathullah, Á. F. García-Fernández, and L. Svensson, "Generalized optimal sub-pattern assignment metric," in Proceedings of the 20th International Conference on Information Fusion, Xi'an, China, 2017.

\section{APPENDIX A \\ LINEAR GAUSSIAN PMBM FILTER RECURSION}

Assuming linear Gaussian dynamics and linear measurements according to

$$
\begin{aligned}
p_{k+1, k}\left(x \mid x^{\prime}\right) & =\mathcal{N}\left(x ; F x^{\prime}, Q\right), \\
p(z \mid x) & =\mathcal{N}(z ; H x, R),
\end{aligned}
$$

constant detection probability $p_{\mathrm{D}}(x)=p_{\mathrm{D}}$, and constant survival probability $p_{\mathrm{S}}(x)=p_{\mathrm{S}}$, the prediction and update steps of the PMBM filter are given as follows.

\section{A. Prediction}

Let the posterior PMBM density be defined by the set of parameters

$$
\lambda_{k \mid k}^{\mathrm{u}},\left\{w_{k \mid k}^{j},\left\{r_{k \mid k}^{j, i}, p_{k \mid k}^{j, i}\right\}_{i \in \mathbb{I}_{k \mid k}^{j}}\right\}_{j \in \mathbb{J}_{k \mid k}},
$$

where the intensity of undetected targets $\lambda^{\mathrm{u}}$ is a Gaussian mixture

$$
\lambda_{k \mid k}^{\mathrm{u}}(x)=\sum_{i=1}^{N^{\mathrm{u}}} w_{k \mid k}^{\mathrm{u}, i} \mathcal{N}\left(x ; \hat{x}_{k \mid k}^{\mathrm{u}, i}, P_{k \mid k}^{\mathrm{u}, i}\right),
$$

and the spatial density of the $j$ th Bernoulli component in the $i$ th global hypothesis is Gaussian distributed according to $p_{k \mid k}^{j, i}(x)=\mathcal{N}\left(x ; \hat{x}_{k \mid k}^{j, i}, P_{k \mid k}^{j, i}\right)$. Then, with single-target transition model given by (42a) and Gaussian mixture birth intensity according to

$$
\lambda_{k}^{\mathrm{b}}(x)=\sum_{i=1}^{N^{\mathrm{b}}} w_{k}^{\mathrm{b}, i} \mathcal{N}\left(x ; \hat{x}_{k}^{\mathrm{b}, i}, P_{k}^{\mathrm{b}, i}\right),
$$

the predicted PMBM density is on the same form as (43). The predicted intensity of undetected targets is given by

$$
\begin{aligned}
\lambda_{k+1 \mid k}^{\mathrm{u}}(x) & =\sum_{i=1}^{N^{\mathrm{b}}} w_{k+1}^{\mathrm{b}, i} \mathcal{N}\left(x ; \hat{x}_{k+1}^{\mathrm{b}, i}, P_{k+1}^{\mathrm{b}, i}\right) \\
& +\sum_{i=1}^{N^{\mathrm{u}}} w_{k+1 \mid k}^{\mathrm{u}, i} \mathcal{N}\left(x ; \hat{x}_{k+1 \mid k}^{\mathrm{u}, i}, P_{k+1 \mid k}^{\mathrm{u}, i}\right),
\end{aligned}
$$

where

$$
\begin{aligned}
w_{k+1 \mid k}^{\mathrm{u}, i} & =p_{\mathrm{s}} w_{k \mid k}^{\mathrm{u}, i}, \\
\hat{x}_{k+1 \mid k}^{\mathrm{u}, i} & =F \hat{x}_{k \mid k}^{\mathrm{u}, i}, \\
P_{k+1 \mid k}^{\mathrm{u}, i} & =F P_{k \mid k}^{\mathrm{u}, i} F^{\top}+Q,
\end{aligned}
$$

the Bernoulli components are predicted according to

$$
\begin{aligned}
r_{k+1 \mid k}^{j, i} & =p_{\mathrm{s}} r_{k \mid k}^{j, i}, \\
\hat{x}_{k+1 \mid k}^{j, i} & =F \hat{x}_{k \mid k}^{j, i}, \\
P_{k+1 \mid k}^{j, i} & =F P_{k \mid k}^{j, i} F^{\boldsymbol{\top}}+Q,
\end{aligned}
$$

and $w_{k+1 \mid k}^{j}=w_{k \mid k}^{j}, \mathbb{I}_{k+1 \mid k}^{j}=\mathbb{I}_{k \mid k}^{j}, \mathbb{J}_{k+1 \mid k}=\mathbb{J}_{k \mid k}$. 


\section{B. Measurement update}

1) Data association: As the true origins of measurements are unknown, association hypotheses are required. Let $\mathbb{M}$ be an index set for the elements of the measurement set $Z$, i.e.,

$$
Z=\left\{z^{m}\right\}_{m \in \mathbb{M}}
$$

and let $\mathcal{A}^{j}$ be a collection of all possible association hypotheses $A$ for the $j$ th global hypothesis, i.e., the $j$ th $\mathrm{MB}$, of which the targets are indexed by $\mathbb{I}^{j}$. Then, an association hypothesis $A \in \mathcal{A}^{j}$ is a partition of $\mathbb{M} \cup \mathbb{I}^{j}$ into nonempty disjoint subsets $C \in A$, called index cells [14].

The standard assumptions in multi-target tracking that the targets are independent of each other implies that an index cell contains at most one target index and at most one measurement index, i.e., $\left|C \cap \mathbb{I}^{j}\right| \leq 1$ and $|C \cap \mathbb{M}| \leq 1$ for all $C \in A \in \mathcal{A}^{j}$. In the following, let $i_{C}$ and $m_{C}$ denote the target and measurement indices corresponding to index cell $C$.

2) Update equations: Let the predicted PMBM density be defined by the set of parameters

$$
\lambda_{k \mid k-1}^{\mathrm{u}},\left\{w_{k \mid k-1}^{j},\left\{r_{k \mid k-1}^{j, i}, p_{k \mid k-1}^{j, i}\right\}_{i \in \mathbb{I}_{k \mid k-1}^{j}}\right\}_{j \in \mathbb{J}_{k \mid k-1}}
$$

where the intensity of undetected targets $\lambda^{\mathrm{u}}$ is a Gaussian mixture

$$
\lambda_{k \mid k-1}^{\mathrm{u}}(x)=\sum_{i=1}^{N^{\mathrm{u}}} w_{k \mid k-1}^{\mathrm{u}, i} \mathcal{N}\left(x ; \hat{x}_{k \mid k-1}^{\mathrm{u}, i}, P_{k \mid k-1}^{\mathrm{u}, i}\right),
$$

and the spatial density of Bernoulli component $j, i$ is Gaussian distributed according to $p_{k \mid k-1}^{j, i}(x)=\mathcal{N}\left(x ; \hat{x}_{k \mid k-1}^{j, i}, P_{k \mid k-1}^{j, i}\right)$. Then, with a set of measurements $Z$ and single-measurement likelihood according to (42b), the updated PMBM density is a PMBM density given by

$$
\begin{aligned}
\pi^{\mathrm{PMBM}}(X \mid Z) & =\sum_{X^{\mathrm{u} \uplus X^{\mathrm{d}}}=X} \pi^{\mathrm{P}}\left(X^{\mathrm{u}}\right) \pi^{\mathrm{MBM}}\left(X^{\mathrm{d}}\right), \\
\pi^{\mathrm{P}}\left(X^{\mathrm{u}}\right) & =e^{-\left\langle\lambda_{k \mid k}^{\mathrm{u}}, 1\right\rangle} \prod_{x \in X^{\mathrm{u}}} \lambda_{k \mid k}^{\mathrm{u}}(x), \\
\pi^{\mathrm{MBM}}\left(X^{\mathrm{d}}\right) & =\sum_{j \in \mathbb{J}_{k \mid k-1}} \sum_{A \in \mathcal{A}^{j}} w_{A}^{j} \pi_{A}^{j}\left(X^{\mathrm{d}}\right), \\
\pi_{A}^{j}\left(X^{\mathrm{d}}\right) & =\sum_{\uplus_{C} \in A} \prod_{X^{i} C=X^{\mathrm{d}}} \prod_{C \in A} \pi_{C}^{j}\left(X^{i_{C}}\right),
\end{aligned}
$$

where the weights of the global hypotheses are given by

$$
w_{A}^{j}=\frac{w_{k \mid k-1}^{j} \prod_{C \in A} \mathcal{L}_{C}}{\sum_{j \in \mathbb{J}_{k \mid k-1}} \sum_{A \in \mathcal{A}^{j}} w_{k \mid k-1}^{j} \prod_{C \in A} \mathcal{L}_{C}}
$$

and the parameters of (52) and (53) are described in the following.

a) Intensity of undetected targets: The updated intensity of undetected targets $\lambda_{k \mid k}^{\mathrm{u}}$ is a Gaussian mixture, with weights updated according to

$$
w_{k \mid k}^{\mathrm{u}, i}=p_{\mathrm{D}} w_{k \mid k-1}^{\mathrm{u}, i}
$$

and unchanged mixture components, i.e., $\hat{x}_{k \mid k}^{\mathrm{u}, i}=\hat{x}_{k \mid k-1}^{\mathrm{u}, i}$ and $P_{k \mid k}^{\mathrm{u}, i}=P_{k \mid k-1}^{\mathrm{u}, i}$. b) Potential target detected for the first time: Each measurement $z_{k}^{m}$ generates a new Bernoulli component with existence probability and density according to

$$
\begin{aligned}
r_{k \mid k} & =\frac{e_{k}\left(z_{k}^{m}\right)}{\lambda^{\mathrm{fa}}\left(z^{m}\right)+e_{k}\left(z_{k}^{m}\right)} \\
p(x) & =\frac{1}{e_{k}\left(z_{k}^{m}\right)} \sum_{i=1}^{N^{\mathrm{u}}} c_{k}^{\mathrm{u}, i} \mathcal{N}\left(x ; \hat{x}_{k \mid k}^{\mathrm{u}, i}, P_{k \mid k}^{\mathrm{u}, i}\right)
\end{aligned}
$$

where

$$
\begin{aligned}
\hat{x}_{k \mid k}^{\mathrm{u}, i} & =\hat{x}_{k \mid k-1}^{\mathrm{u}, i}+K_{k}\left(z_{k}^{m}-H \hat{x}_{k \mid k-1}^{\mathrm{u}, i}\right) \\
P_{k \mid k}^{\mathrm{u}, i} & =P_{k \mid k-1}^{\mathrm{u}, i}-K_{k} H P_{k \mid k-1}^{\mathrm{u}, i} \\
K_{k} & =P_{k \mid k-1}^{\mathrm{u}, i} H^{\top}\left(S_{k}^{\mathrm{u}, i}\right)^{-1} \\
S_{k}^{\mathrm{u}, i} & =H P_{k \mid k-1}^{\mathrm{u}, i} H^{\top}+R \\
c_{k}^{\mathrm{u}, i} & =p_{\mathrm{D}} w_{k \mid k-1}^{\mathrm{u}, i} \mathcal{N}\left(z_{k}^{m} ; H \hat{x}_{k \mid k-1}^{\mathrm{u}, i}, S_{k}^{\mathrm{u}, i}\right) \\
e_{k}\left(z_{k}^{m}\right) & =\sum_{i=1}^{N^{\mathrm{u}}} c_{k}^{\mathrm{u}, i}
\end{aligned}
$$

The Gaussian mixture in (55b) is approximated as a Gaussian distribution by performing moment matching. The likelihood corresponding to the hypothesis that measurement $z_{k}^{m}$ originates from a previously undetected target is given by

$$
\mathcal{L}_{C}=\lambda^{\mathrm{fa}}\left(z^{m}\right)+e_{k}\left(z_{k}^{m}\right)
$$

with $C \cap \mathbb{I}_{k \mid k-1}^{j}=\emptyset$ and $C \cap \mathbb{M}=m$.

c) Previously detected targets: A predicted Bernoulli component with existence probability $r_{k \mid k-1}^{j, i}$ and spatial density $p_{k \mid k-1}^{j, i}(x)=\mathcal{N}\left(x ; \hat{x}_{k \mid k-1}^{j, i}, P_{k \mid k-1}^{j, i}\right)$ generates a misdetection hypothesis and one hypothesis for each measurement in $Z$.

Under the hypothesis that the target is misdetected, the updated existence probability is given by

$$
r_{k \mid k}=\frac{r_{k \mid k-1}^{j, i}\left(1-p_{\mathrm{D}}\right)}{1-r_{k \mid k-1}^{j, i}+r_{k \mid k-1}^{j, i}\left(1-p_{\mathrm{D}}\right)}
$$

and the density remains the same, i.e., $\hat{x}_{k \mid k}^{j, i}=\hat{x}_{k \mid k-1}^{j, i}$ and $P_{k \mid k}^{j, i}=P_{k \mid k-1}^{j, i}$. The likelihood corresponding to this hypothesis is given by

$$
\mathcal{L}_{C}=1-r_{k \mid k-1}^{j, i}+r_{k \mid k-1}^{j, i}\left(1-p_{\mathrm{D}}\right)
$$

with $C \cap \mathbb{I}_{k \mid k-1}^{j}=i$ and $C \cap \mathbb{M}=\emptyset$.

Under the hypothesis that the target is detected with measurement $z_{k}^{m}$, the Bernoulli component has existence probability $r_{k \mid k}=1$ and density $\mathcal{N}\left(x ; \hat{x}_{k \mid k}^{j, i}, P_{k \mid k}^{j, i}\right)$ where

$$
\begin{aligned}
\hat{x}_{k \mid k}^{j, i} & =\hat{x}_{k \mid k-1}^{j, i}+K_{k}\left(z_{k}^{m}-H \hat{x}_{k \mid k-1}^{j, i}\right) \\
P_{k \mid k}^{j, i} & =P_{k \mid k-1}^{j, i}-K_{k} H P_{k \mid k-1}^{j, i} \\
K_{k} & =P_{k \mid k-1}^{j, i} H^{\top}\left(S_{k}^{j, i, m}\right)^{-1} \\
S_{k}^{j, i, m} & =H P_{k \mid k-1}^{j, i} H^{\top}+R
\end{aligned}
$$

The likelihood corresponding to this hypothesis is given by

$$
\mathcal{L}_{C}=r_{k \mid k-1}^{j, i} p_{\mathrm{D}} \mathcal{N}\left(z^{m} ; H \hat{x}_{k \mid k-1}^{j, i}, S_{k \mid k-1}^{j, i, m}\right)
$$

with $C \cap \mathbb{I}_{k \mid k-1}^{j}=i$ and $C \cap \mathbb{M}=m$. 


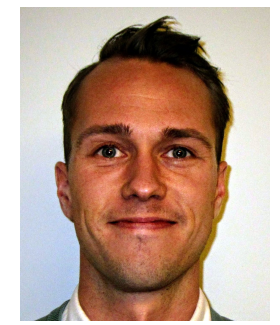

Per Boström-Rost received his M.Sc. in Applied Physics and Electrical Engineering in 2013 and his Ph.D. in Electrical Engineering in 2021, both from Linköping University.

Dr. Boström-Rost is currently working as a systems engineer at Saab Aeronautics, Linköping, Sweden. His main research interests include sensor fusion and sensor management with applications to target tracking.

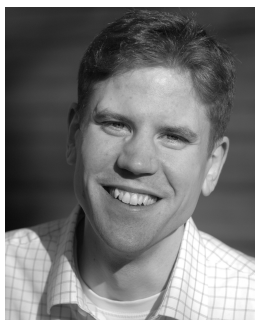

Daniel Axehill received his M.Sc. degree in Applied Physics and Electrical Engineering in 2003. Furthermore, he received the degree of Lic.Eng. in Automatic Control in 2005 and the Ph.D. degree in Automatic Control in 2008. All three degrees are from Linköping University, Linköping, in Sweden. In year 2006 he spent three months at UCLA in Los Angeles. From January 2009 and until November 2010 he was a post-doc at the Automatic Control Laboratory at ETH Zurich. He is currently employed as an Associate Professor at the Division of Automatic Control at Linköping University. His research interests are related to optimization, optimal control, motion planning, hybrid systems, and applications of control.

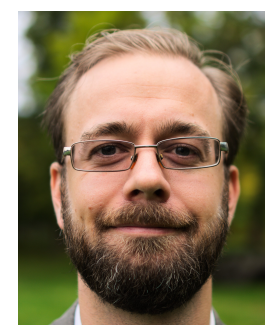

Gustaf Hendeby (S'04-M'09-SM'17) received his M.Sc. in Applied Physics and Electrical Engineering in 2002 and his Ph.D. in Automatic Control in 2008, both from Linköping University, Linköping, Sweden.

$\mathrm{He}$ is Associate Professor and Docent in the division of Automatic Control, Department of Electrical Engineering, Linköping University. He worked as Senior Researcher at the German Research Center for Artificial Intelligence (DFKI) 2009-2011, and Senior Scientist at Swedish Defense Research Agency (FOI) and held an adjunct Associate Professor position at Linköping University 2011-2015. Dr. Hendeby's main research interests are stochastic signal processing and sensor fusion with applications to nonlinear problems, target tracking, and simultaneous localization and mapping (SLAM). He has experience of both theoretical analysis as well as implementation aspects.

Dr. Hendeby is since 2018 an Associate Editor for IEEE Transactions on Aerospace and Electronic Systems. 